\title{
Thermal properties of TRIM-GMA copolymers with pendant amine groups
}

\author{
M. Maciejewska ${ }^{1}$
}

Received: 7 March 2016/ Accepted: 7 June 2016/Published online: 20 June 2016

(c) The Author(s) 2016. This article is published with open access at Springerlink.com

\begin{abstract}
The porous copolymers of glycidyl methacrylate crosslinked with trimethylolpropane trimethacrylate were prepared in the form of regular microspheres by suspension-emulsion polymerization. In order to obtain adsorbents bearing functional groups, the porous methacrylate network was modified by subsequent reaction with diethylenetriamine. The thermal behavior of the obtained material was studied using TG and DSC. It was found that the process of modification considerably changed the textural and thermal properties of the polymers.
\end{abstract}

Keywords Porous polymers $\cdot$ Microstructure $\cdot$ Thermal properties $\cdot$ TG $\cdot$ DSC

\section{List of symbols}

$\begin{array}{ll}\delta & \text { Solubility parameter/(MPa) }{ }^{1 / 2} \\ D_{\mathrm{BJH}} & \text { Pore diaameter } / \AA \\ \text { FDT } & \text { Temperature of final decomposition } /{ }^{\circ} \mathrm{C} \\ \text { IDT } & \text { Initial decomposition temperature } /{ }^{\circ} \mathrm{C} \\ S_{\text {BET }} & \text { Specific surface area } / \mathrm{m}^{2} \mathrm{~g}^{-1} \\ T_{1 \mathrm{max}} & \text { Temperature of the first maximum rate of } \\ & \text { mass loss } /{ }^{\circ} \mathrm{C} \\ T_{2 \max } & \text { Temperature of the second maximum rate } \\ & \text { of mass loss } /{ }^{\circ} \mathrm{C} \\ T_{20} \% & \text { Temperature of } 20 \% \text { mass loss } /{ }^{\circ} \mathrm{C} \\ T_{50} \% & \text { Temperature of } 50 \% \text { mass loss } /{ }^{\circ} \mathrm{C} \\ \text { TRIM- } & \text { The parent copolymer, molar ratio of } \\ \text { GMA1 } & \text { trimethylolpropane trimethacrylate to } \\ & \text { glycidyl methacrylate equal } 1: 1\end{array}$

M. Maciejewska

mmacieje@umcs.pl

1 Faculty of Chemistry, Maria Curie-Skłodowska University, pl. M. Curie-Skłodowskiej 3, 20-031 Lublin, Poland
TRIM-

GMA2

TRIM-

GMA3

TRIM-

GMA4

TRIM-

GMA5

TRIM-

GMA1+A

TRIM-

GMA2+A

TRIM-

GMA3+A

TRIM-

GMA4+A

TRIM-

GMA5+A

V
The parent copolymer, molar ratio of trimethylolpropane trimethacrylate to glycidyl methacrylate equal 1:2

The parent copolymer, molar ratio of trimethylolpropane trimethacrylate to glycidyl methacrylate equal 1:3

The parent copolymer, molar ratio of trimethylolpropane trimethacrylate to glycidyl methacrylate equal 1:4

The parent copolymer, molar ratio of trimethylolpropane trimethacrylate to glycidyl methacrylate equal 1:5 The copolymer modified with diethylenetriamine, molar ratio of trimethylolpropane trimethacrylate to glycidyl methacrylate equal 1:1 The copolymer modified with diethylenetriamine, molar ratio of trimethylolpropane trimethacrylate to glycidyl methacrylate equal 1:2 The copolymer modified with diethylenetriamine, molar ratio of trimethylolpropane trimethacrylate to glycidyl methacrylate equal 1:3 The copolymer modified with diethylenetriamine, molar ratio of trimethylolpropane trimethacrylate to glycidyl methacrylate equal 1:4 The copolymer modified with diethylenetriamine, molar ratio of trimethylolpropane trimethacrylate to glycidyl methacrylate equal 1:5 Pore volume $/ \mathrm{cm}^{3} \mathrm{~g}^{-1}$ 


\section{Introduction}

Porous polymers in the form of microspheres are among the most effective material for many separation processes. In comparison with the commonly used adsorbents based on silica gel, polymers are stable throughout the whole $\mathrm{pH}$ range, and they can be easily functionalized and have the ability to create specific sorption spaces. In their structure, they combine the most desirable characteristics of porous materials and polymers and consequently are used as various kinds of sorbents [1-9]. In the case of specific sorption processes, sorbents should possess functional groups on their surface. Over past years, copolymers based on glycidyl methacrylate (GMA) have attracted growing attention. The presence of epoxy groups allows a number of chemical modifications on the initial polymer to suit a variety of applications.

Consequently, preparation of GMA-based copolymers and their modifications is the subject of numerous papers [10-22]. The conducted studies had indicated that an amine group is one of the most efficient functional groups for heavy metal ion removal. Functionalization of GMA copolymers with amines provides desirable sorbents with high capacity, fast kinetics and good selectivity for the heavy metal ions. Interestingly, the type of amine significantly affects the selectivity as well as porosity parameters of the functionalized copolymers. However, some properties of GMA-based copolymers, such as thermal stability and poor physical performance, need to be improved. Synthesis of GMA-based macroporous microspheres of high degree of crosslinking can considerably improve the chemical, physical and thermal properties of the functionalized copolymers. Recently, the synthesis and some properties of porous microspheres of glycidyl methacrylate (GMA) crosslinked with trimethylolpropane trimethacrylate (TRIM) modified with diethylenetriamine have been described [23]. It was found out that the modified copolymers show considerable affinity toward metal ions and can be used as effective sorbents for the removal of $\mathrm{Cu}(\mathrm{II}), \mathrm{Zn}(\mathrm{II}), \mathrm{Cd}(\mathrm{II})$ and $\mathrm{Pb}(\mathrm{II})$.

This study presents the detailed investigation of thermal properties of the parent and modified copolymers. They were evaluated by the means of TG and DSC. Additionally, the textural characterization was carried out on the basis of the low-temperature nitrogen adsorption.

\section{Experimental}

\section{Chemicals}

2,3-Epoxypropyl methacrylate (GMA) and trimethylolpropane trimethacrylate (TRIM) (Sigma-Aldrich, Steinheim, Germany) were washed with $5 \%$ aqueous sodium hydroxide in order to remove inhibitors. Bis(2-ethylhexyl) sulfosuccinate sodium salt (DAC,BP) and $\alpha, \alpha^{\prime}$-azoisobutyronitrile (AIBN) purchased from Fluka AG (Buchs, Switzerland) were used without purification. Toluene, $n$ dodecane, acetone and methanol (reagent grade) were from POCh (Gliwice, Poland). Diethylenetriamine was purchased from Sigma-Aldrich.

\section{Preparation of the GMA-TRIM microspheres}

Copolymerization was performed in an aqueous suspension medium. Distilled water $(195 \mathrm{~mL})$ and $2.2 \mathrm{~g}$ of bis(2ethylhexyl)sulfosuccinate sodium salt were stirred for $2 \mathrm{~h}$ at $80{ }^{\circ} \mathrm{C}$ in order to dissolve the surfactant. Then, the solution containing $15 \mathrm{~g}$ of monomers (GMA and TRIM) and $0.2 \mathrm{~g}$ of $\alpha, \alpha^{\prime}$-azoisobutyronitrile dissolved in $22.5 \mathrm{~mL}$ of toluene was prepared and added while stirring to the aqueous medium. Molar ratios of GMA to TRIM were changed from 1:1 to 5:1. Copolymerization was performed for $20 \mathrm{~h}$ at $80^{\circ} \mathrm{C}$. Porous beads formed in this process were collected by filtration, and an extensive cleaning procedure was applied in order to remove the diluent unreacted monomers and physically adsorbed stabilizer. The cleaning process was as follows: the microspheres were separated from the aqueous phase by filtration of the polymerization mixture using $5-\mu \mathrm{m}$ filter paper. The microspheres were first washed with water, and the polymeric aggregates were removed by sieving. The microspheres were dispersed in water, and the dispersion was sonificated for $0.5 \mathrm{~h}$ in an ultrasonic bath. Next, the water phase was removed and the microspheres were resuspended in methyl alcohol. This dispersion was sonificated for $1 \mathrm{~h}$. Methyl alcohol was removed, and the microspheres were transferred into toluene and were kept there by stirring about $0.5 \mathrm{~h}$. Then, the toluene was removed and microspheres were stirred with methyl alcohol for about $0.5 \mathrm{~h}$. Methyl alcohol was removed, and the microspheres were washed with distilled water, collected by filtration and dried at reduced pressure at $65^{\circ}$ for $48 \mathrm{~h}$.

\section{Modification of the epoxy groups}

The epoxy groups present in the copolymer were modified by the reaction with diethylenetriamine (Fig. 1). The procedure is as follows: in a $250-\mathrm{cm}^{3}$ round-bottomed twonecked flask equipped with a mechanical stirrer and a thermometer, $10 \mathrm{~g}$ of selected beads was placed together with DETA and $200 \mathrm{~mL}$ of toluene, and the whole was heated on a water bath at $80{ }^{\circ} \mathrm{C}$ for $24 \mathrm{~h}$. The modified beads obtained were washed with distilled water, collected by filtration, dried and extracted in a Soxhlet apparatus with boiling toluene for $5 \mathrm{~h}$. The product was then dried under reduced pressure at $30{ }^{\circ} \mathrm{C}$. 
<smiles>C=C(C)C(=O)OCC(CC)(COC(=O)C(=C)C)COC(=O)C(=C)C</smiles>

GMA

TRIM
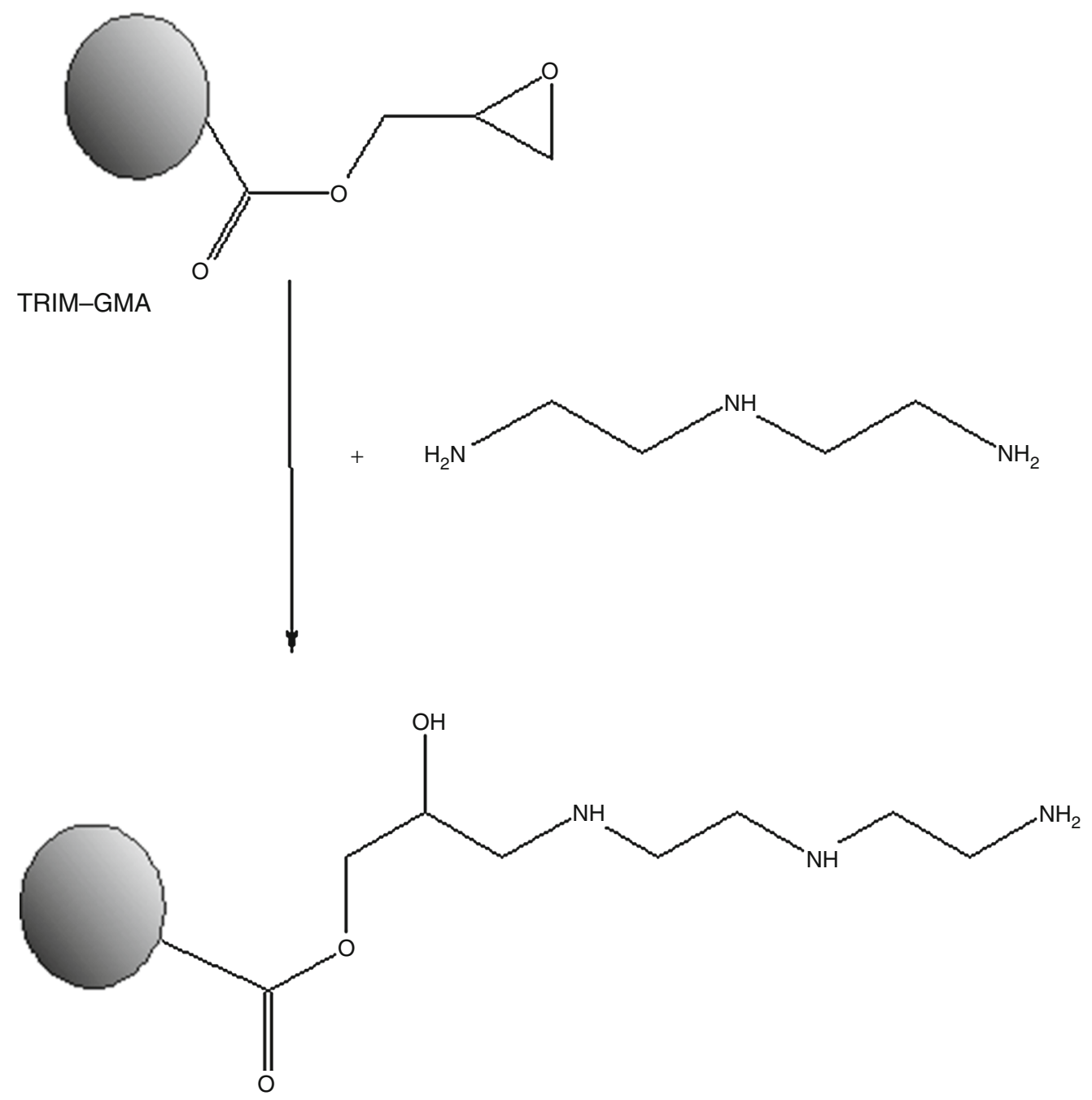

TRIM-GMA + A

Fig. 1 Scheme of modification of poly(glycidyl methacrylate-co-trimethylolpropane trimethacrylate) with diethylenetriamine

\section{Methods of analysis}

Textural characterization of the copolymers was carried out using the low-temperature nitrogen adsorption-desorption method. Nitrogen adsorption-desorption isotherms were obtained at liquid nitrogen temperature using a volumetric adsorption analyzer ASAP 2405 (Micromeritics Inc., USA). The measurements of the porous structure of the 
copolymers were preceded by outgassing of the samples at $140{ }^{\circ} \mathrm{C}$ for $2 \mathrm{~h}$. The specific surface area of the investigated samples was calculated using the Brunauer-Emmet-Teller (BET) method for the adsorption data in the range of a relative pressure $p / p_{\mathrm{o}} 0.05-0.25$. The total pore volume was estimated from a single-point adsorption at a relative pressure of 0.985 . The pore size was obtained from the desorption branch of the isotherm using the Barrett-Joyner-Halenda $(\mathrm{BJH})$ procedure.

The surface of the beads was also examined using an atomic LEO 1430 VP numerical scanning electron microscope (Germany) with a countershaft and an energy-dispersive X-ray detector.

The thermal properties of the synthesized composites were evaluated on the basis of measurements taken using the STA449, F1 Jupiter analyzer from Netzsch (Günzbung, Germany). The procedure was as follows: about $10 \mathrm{mg}$ of the sample was placed in the TG pan and heated in helium or in air atmosphere at a rate of $10 \mathrm{~K} \mathrm{~min}^{-1}$ up to $1000{ }^{\circ} \mathrm{C}$ with a sample mass of about $10 \mathrm{mg}$. The initial decomposition temperature (IDT), $T_{20} \%$ and $T_{50} \%$ of mass loss and final decomposition temperature (FDT) were determined.

The gaseous decomposition products formed under the degradation of functionalized copolymers were analyzed by means of a FTIR spectrometer TGA 585 Bruker (Germany) coupled online to a STA instrument by a Teflon transfer line. An FTIR spectrum was gathered every $10^{\circ} \mathrm{C}$ in the spectral range from 600 to $4000 \mathrm{~cm}^{-1}$ with a resolution of $4 \mathrm{~cm}^{-1}$.

\section{Results and discussion}

Porous copolymers of poly(TRIM-co-GMA) used in this study were synthesized in the form of regular microspheres according procedure described in detail elsewhere [24]. In the next step, a reaction with diethylenetriamine was conducted. Epoxy groups present in the copolymer matrix were opened, and porous copolymers with pendant amine groups were generated. The newly obtained copolymers differ considerably from the parent ones in terms of porous structure parameters as well as thermal properties. The modification process leads to a noticeable decrease in the value of specific surface area (Table 1). This effect is especially visible in the case of TRIM-GMA1 copolymer. After reaction with diethylenetriamine, the value of surface area diminishes to $203 \mathrm{~m}^{2} \mathrm{~g}^{-1}$, whereas the value of surface area for parent copolymer is $333 \mathrm{~m}^{2} \mathrm{~g}^{-1}$. At the same time, the pendant amine groups contribute to an increase in the pore diameters from 190 to $250 \AA$. For the other copolymers, the changes are not so spectacular but still significant.
Table 1 Basic parameters of porous structure of the parent and modified copolymers

\begin{tabular}{lcll}
\hline Copolymer & $\begin{array}{l}\text { Specific surface area } \\
S_{\mathrm{BET}} / \mathrm{m}^{2} \mathrm{~g}^{-1}\end{array}$ & $\begin{array}{l}\text { Pore volume } \\
V / \mathrm{cm}^{3} \mathrm{~g}^{-1}\end{array}$ & $D_{\mathrm{BJH}} / \AA$ \\
\hline TRIM-GMA1 & 333 & 0.694 & 190 \\
TRIM-GMA2 & 182 & 0.665 & 250 \\
TRIM-GMA3 & 99 & 0.405 & 312 \\
TRIM-GMA4 & 90 & 0.537 & 380 \\
TRIM-GMA5 & 86 & 0.461 & 390 \\
TRIM-GMA1+A & 203 & 0.475 & 250 \\
TRIM-GMA2+A & 150 & 0.634 & 320 \\
TRIM-GMA3+A & 101 & 0.585 & 380 \\
TRIM-GMA4+A & 87 & 0.523 & 400 \\
TRIM-GMA5+A & 81 & 0.627 & 490 \\
\hline
\end{tabular}

The process of modification of the TRIM-GMA copolymers with diethylenetriamine also changes the thermal properties of these materials. TG and DTG curves for parent and modified TRIM-GMA copolymer are presented Figs. 2 and 3. The main parameters evaluated on the basis of these curves determined in helium atmosphere for the whole series of copolymers are presented in Table 2. As can be seen, after the process of modification the IDT temperature is higher for the whole series of copolymers. The differences are even more visible for $T_{20} \%$ and $T_{50 \%}$ temperatures. However, the greatest increase can be observed with reference to final decomposition temperature. For all modified copolymers, FDT considerably exceeds $700{ }^{\circ} \mathrm{C}$. While comparing the different factors that could influence the thermal behavior of the porous TRIMGMA copolymers, it can be seen that the modification process has much greater impact on the thermal properties of the copolymers than molar ratio of the functional monomer to crosslinker.

A detailed analysis of DTG curves (Figs. 2, 3) can provide another piece of information. In the case of parent TRIM-GMA copolymers, two separate peaks are visible. The first maximum can be attributed to the decomposition of epoxy groups and the second to the degradation of ester bonds. After the reaction of the epoxy ring with diethylenetriamine (A), the peaks on the DTG curves are not so clearly separated. Still two peaks can be detected on the DTG curves, but they start to merge.

In the case of analysis conducted in air, the amine groups introduced on the surface of the copolymers contribute to the changes in thermal behavior of the materials under study. Their presence initiates autocatalytic exothermic oxidation of organic layers and consequently decreases the IDT (Table 3 ). A the same time, the $T_{20} \%, T_{50} \%$ and FDT temperatures are much higher in comparison with the values 
Fig. 2 TG curves of parent (TRIM-GMA1) and modified (TRIM-GMA1+A) copolymers determined in helium

Fig. 3 DTG curves of modified copolymers determined in helium TRIM-GMA1+A [1], TRIM-GMA2+A [2], TRIMGMA3+A [3], TRIMGMA4+A [4], TRIMGMA5+A [5]
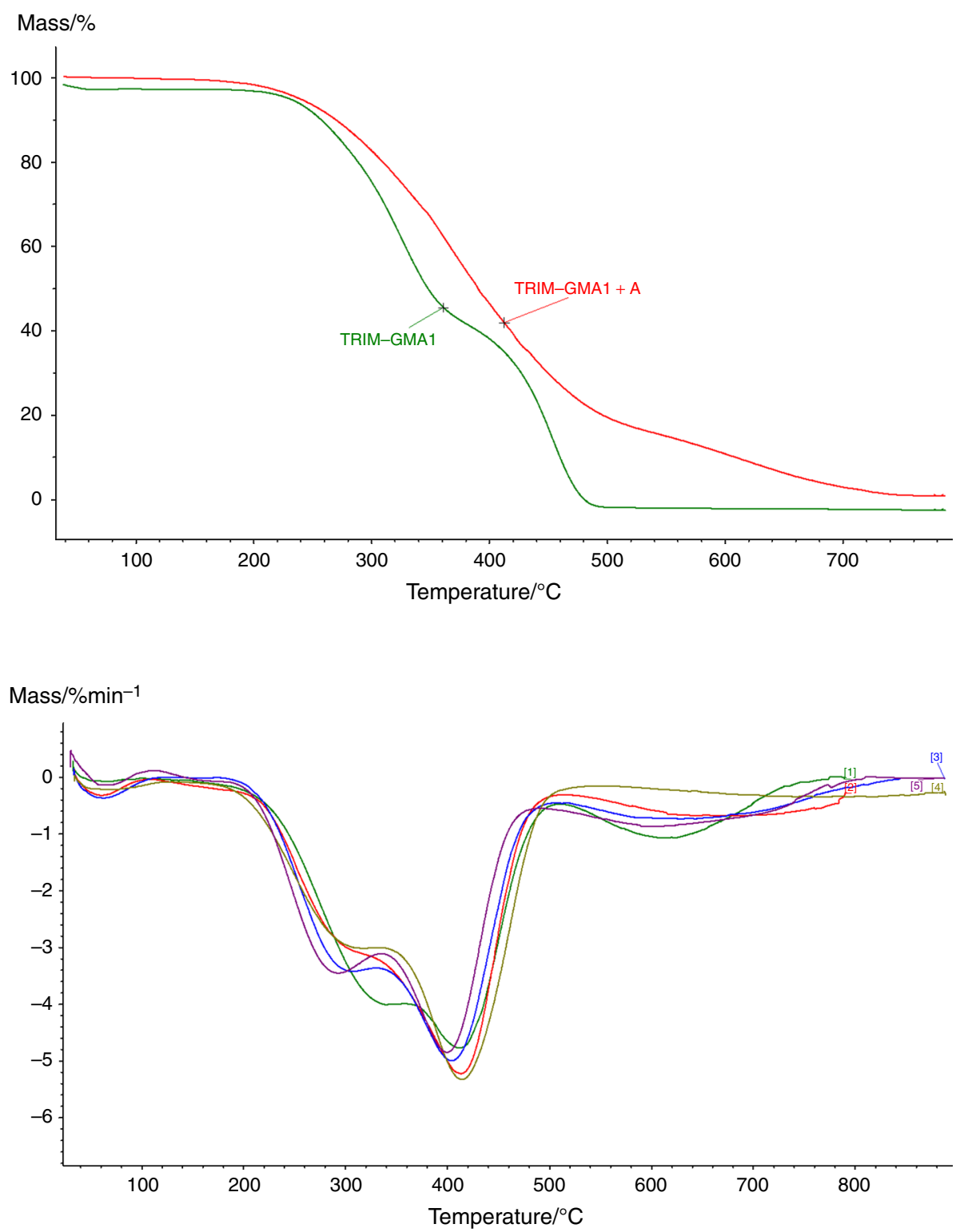

Table 2 Thermal stability of the copolymers under study determined in helium

\begin{tabular}{|c|c|c|c|c|c|c|}
\hline Copolymer & $\mathrm{IDT} /{ }^{\circ} \mathrm{C}$ & $T_{20} \% /{ }^{\circ} \mathrm{C}$ & $T_{50 \%} /{ }^{\circ} \mathrm{C}$ & $\mathrm{FDT} /{ }^{\circ} \mathrm{C}$ & $T_{1 \max } /{ }^{\circ} \mathrm{C}$ & $T_{2 \max } /{ }^{\circ} \mathrm{C}$ \\
\hline TRIM-GMA1 & 219 & 298 & 354 & 487 & 328 & 458 \\
\hline TRIM-GMA2 & 213 & 284 & 332 & 488 & 324 & 456 \\
\hline TRIM-GMA3 & 211 & 266 & 315 & 480 & 298 & 454 \\
\hline TRIM-GMA4 & 212 & 262 & 311 & 480 & 296 & 453 \\
\hline TRIM-GMA5 & 203 & 264 & 319 & 481 & 296 & 418 \\
\hline TRIM-GMA1+A & 237 & 317 & 395 & 720 & 340 & 420 \\
\hline TRIM-GMA2+A & 225 & 311 & 394 & 780 & 305 & 413 \\
\hline TRIM-GMA3+A & 221 & 309 & 393 & 819 & 302 & 407 \\
\hline TRIM-GMA4+A & 220 & 308 & 400 & & 306 & 422 \\
\hline TRIM-GMA5+A & 220 & 300 & 388 & 780 & 293 & 400 \\
\hline
\end{tabular}


for their unmodified counterparts. This fact indicates that the decomposition process involves oxidation.

\section{Volatile decomposition products}

Figure 4 presents an FTIR 3D spectrum of the volatile decomposition products. Moisture vaporization is clearly visible at temperatures ranging from 40 to $235{ }^{\circ} \mathrm{C}$. As the temperature is increased, the formation of additional gaseous products is observed reflecting the beginning of the decomposition processes for the copolymers. The analysis of the chemical structure of GMA and TRIM molecules suggests that the ester bonds present in matrices' networks will decompose at a first stage. Both $\alpha$-hydrogen bond scission (formation and emission of aldehydes) and $\beta$-hydrogen bond scission (formation and emission of carboxylic and vinyl compounds) are possible in the case of GMAderived units [23]. In TRIM-derived units present in networks, only $\beta$-hydrogen bond scission is likely. At the emission spectrum of TRIM-GMA at the first maximum of decomposition (Fig. 5), bands confirming the $\alpha$ - and $\beta$-hydrogen bonds scission are visible, leading to the formation of alkenes (bands at 814 and $936 \mathrm{~cm}^{-1}(\delta=\mathrm{C}-\mathrm{H})$, at $1650 \mathrm{~cm}^{-1}(v \mathrm{C}=\mathrm{C})$ and at $\left.3105 \mathrm{~cm}^{-1}(v=\mathrm{C}-\mathrm{H})\right)$, aldehydes, especially acetaldehyde (bands at $1741 \mathrm{~cm}^{-1}(v$ $\mathrm{C}=\mathrm{O})$ and at $\left.1162 \mathrm{~cm}^{-1}(v \mathrm{C}=\mathrm{O})\right)$, and carboxylic spices (bands at $1790 \mathrm{~cm}^{-1}(v \mathrm{C}=\mathrm{O})$ and $3238 \mathrm{~cm}^{-1}(v \mathrm{O}-$ $\mathrm{H})$. What is more the FTIR spectrum contains vibrations representative of $\mathrm{CO}_{2}\left(2354 \mathrm{~cm}^{-1}\right)$ and $\mathrm{CO}(2171$ and $2120 \mathrm{~cm}^{-1}$ ). Moreover, the evolution of water was confirmed by absorption bands in the region of 3800-3600 and $1600-1400 \mathrm{~cm}^{-1}$. The next maximum of emission of gases produced during the sample decomposition was observed at $450{ }^{\circ} \mathrm{C}$. At this temperature, the crosslinked parts of the sample decompose, and in consequence, significant evolution of carbon dioxide (peaks at 2357, 2311 and $670 \mathrm{~cm}^{-1}$ )

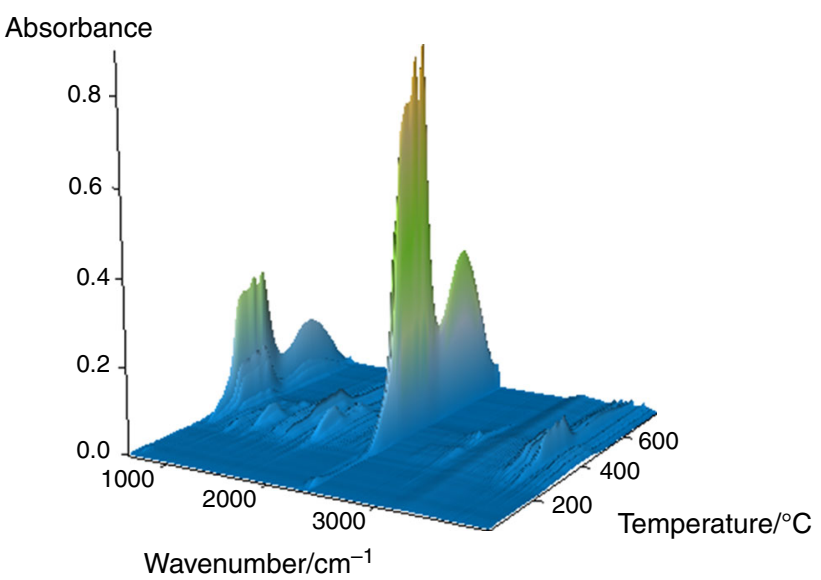

Fig. 4 3D diagram of FTIR spectrum of gases evolved from TRIMGMA copolymer

and carbon monoxide (peaks at 2174, $2120 \mathrm{~cm}^{-1}$ ) connected with decarboxylation processes is observed.

A Gram-Schmidt plot obtained during the thermal decomposition the copolymers under study is presented in Fig. 6. As can be seen, the maximum of the first peak on of the Gram-Schmidt plot corresponds to $T_{2 \max }$ on the DTG curves (Fig. 7). The FTIR spectrum that corresponds to the maximum of the Gram-Schmidt plot is presented in Fig. 5 .

\section{DSC measurements}

The differences in thermal behavior between parent and functionalized copolymers were also observed during DSC measurements (Figs. 8, 9). From the DSC curves, at least three thermal events occur: two exothermic and one endothermic. The exothermic reactions in the thermal treatment of highly crosslinked copolymers could be caused by pendant double bonds. It has been reported that porous copolymers of trimethylolpropane trimethacrylate

Table 3 Thermal stability of the copolymers under study determined in air

\begin{tabular}{|c|c|c|c|c|c|c|}
\hline Copolymer & $\mathrm{IDT} /{ }^{\circ} \mathrm{C}$ & $T_{20} \% /{ }^{\circ} \mathrm{C}$ & $T_{50 \%} /{ }^{\circ} \mathrm{C}$ & $\mathrm{FDT} /{ }^{\circ} \mathrm{C}$ & $T_{1 \text { max }} /{ }^{\circ} \mathrm{C}$ & $T_{2 \max } /{ }^{\circ} \mathrm{C}$ \\
\hline TRIM-GMA1 & 232 & 268 & 344 & 570 & 244 & 358 \\
\hline TRIM-GMA2 & 230 & 249 & 324 & 560 & 245 & 352 \\
\hline TRIM-GMA3 & 225 & 241 & 303 & 560 & 244 & 350 \\
\hline TRIM-GMA4 & 226 & 244 & 301 & 560 & 243 & 350 \\
\hline TRIM-GMA5 & 225 & 242 & 293 & 560 & 240 & 344 \\
\hline TRIM-GMA1+A & 215 & 318 & 392 & 816 & 337 & 409 \\
\hline TRIM-GMA2+A & 200 & 302 & 374 & 736 & 336 & 390 \\
\hline TRIM-GMA3+A & 231 & 290 & 353 & 720 & 260 & 342 \\
\hline TRIM-GMA4+A & 215 & 275 & 330 & 670 & 248 & 315 \\
\hline TRIM-GMA5+A & & & & & & \\
\hline
\end{tabular}


Fig. 5 FTIR spectra of gases evolved from TRIM-GMA copolymer at the maximum of decomposition

Fig. 6 Gram-Schmidt plot obtained during the thermal decomposition the copolymers under study TRIM-GMA1+A [1], TRIM-GMA2+A [2], TRIM-GMA3+A [3], TRIMGMA4+A [4], TRIMGMA5+A [5]

Fig. 7 Gram-Schmidt plot [1] and DTG curve [2] for TRIMGMA1+A copolymer
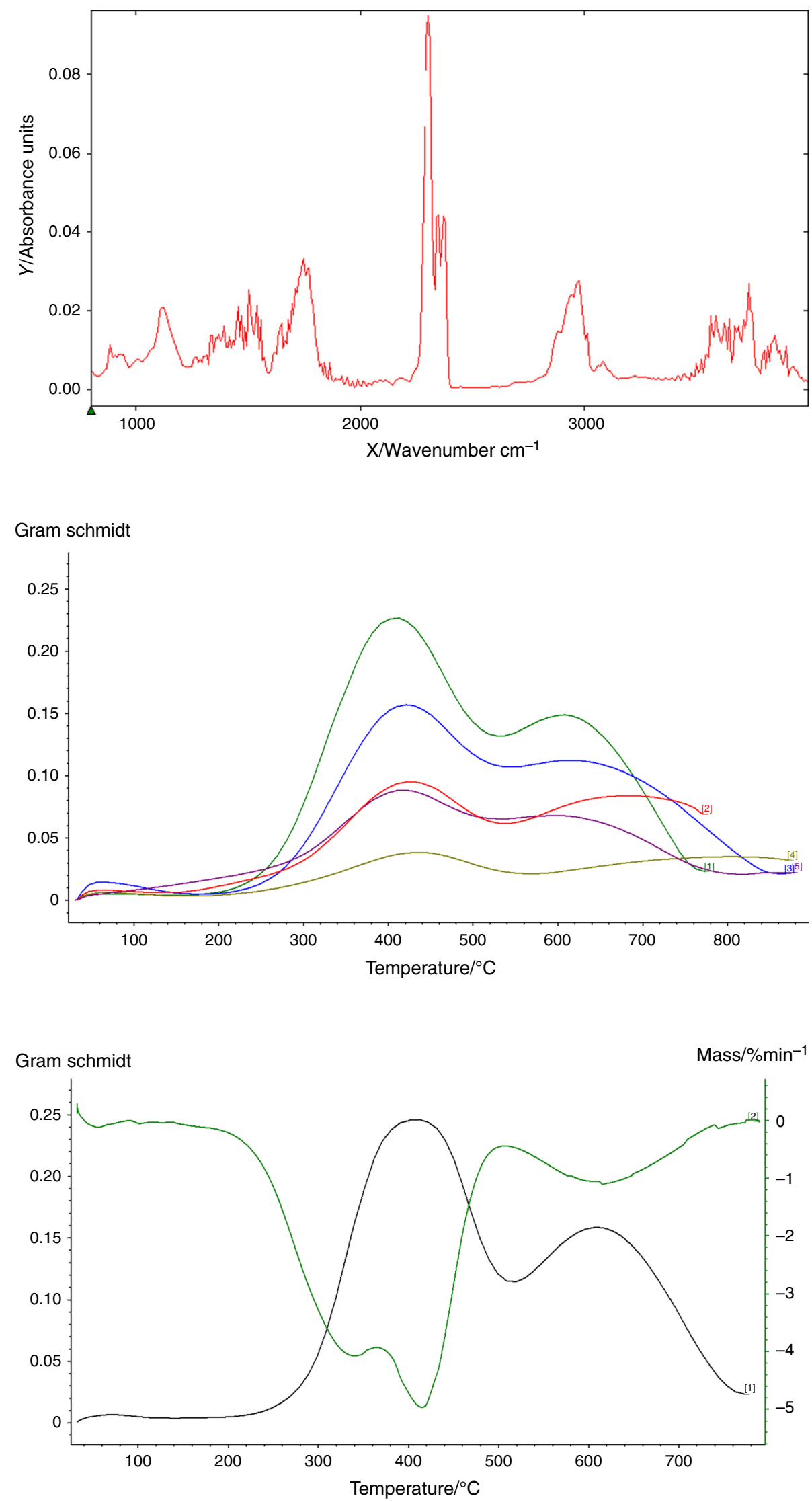
Fig. 8 DSC curves of the parent copolymers determined in helium TRIM-GMA1 [1], TRIM-GMA2 [2], TRIMGMA3 [3], TRIM-GMA4 [4], TRIM-GMA5 [5]
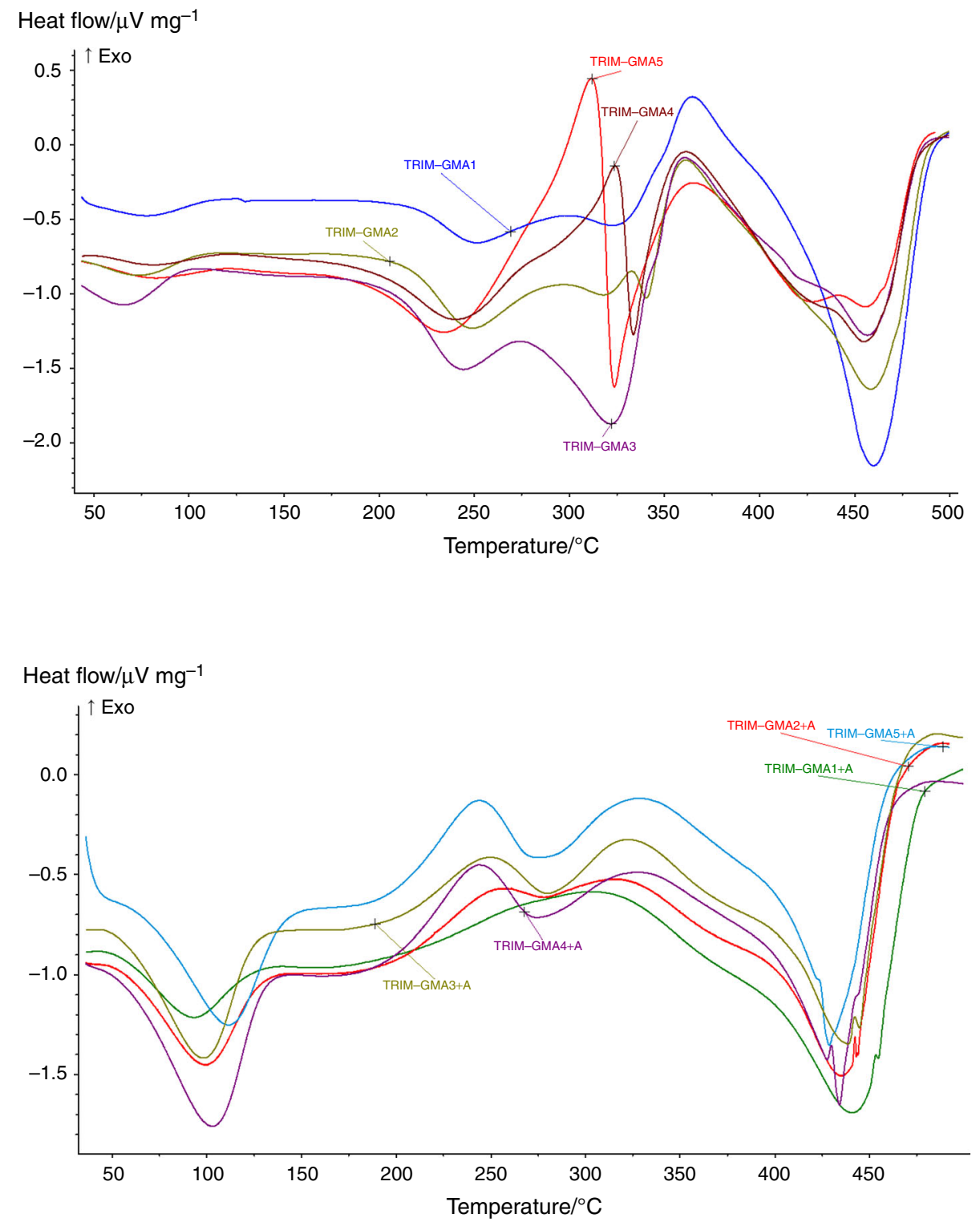

Fig. 9 DSC curves of the modified copolymers determined in helium TRIMGMA1+A [1], TRIMGMA2+A [2], TRIMGMA3+A [3], TRIMGMA4+A [4], TRIMGMA5+A [5] contain a considerable level of unreacted double bonds [25].They can undergo reaction of thermal polymerization as well as double bonds oxidation. What is more, exothermic reaction of thermal crosslinking of the epoxide groups with the carbonyl group of trimethylolpropane trimethacrylate can also occur. In the case of copolymers which contain a significant level of epoxide groups, distinct exothermic peaks at $318{ }^{\circ} \mathrm{C}$ for TRIM-GMA4 and $312^{\circ} \mathrm{C}$ for TRIM-GMA5 are clearly visible in the DSC curves. The endothermic peaks above $450{ }^{\circ} \mathrm{C}$ are associated with the thermal degradation of the copolymers.

After the modification with diethylenetriamine, the DSC curves for the copolymers are considerably changed. First of all, in the case of modified copolymers a distinct endothermic peak at about $100{ }^{\circ} \mathrm{C}$ can be seen. It can be attributed to the process of moisture vaporization from the studied materials.
The presence of amino groups on the surface of the modified copolymers enhances the adsorption of water. The pendant amine groups can also undergo thermal crosslinking with epoxide groups still present in the structure of the copolymers. As a result, exothermic peaks at about $250{ }^{\circ} \mathrm{C}$ for nearly all copolymers are observed. Only in the case of TRIM-GMA1+A copolymer which possesses the minute amount of epoxide and amine groups, this exothermic peak is not observed. The DSC curves for all the copolymers contain an exothermic peak with a maximum at about $330{ }^{\circ} \mathrm{C}$ can be noticed. This can be attributed to the autocatalytic exothermic oxidation of the organic layers.

An endothermic peak is present in the curve above $450{ }^{\circ} \mathrm{C}$. This reflects the thermal degradation of the crosslinked parts of the copolymers. 


\section{Conclusions}

Porous copolymers of glycidyl methacrylate crosslinked with trimethylolpropane trimethacrylate were synthesized in the form of microspheres. In the next step, the reactive epoxy groups were modified by subsequent reaction with diethylenetriamine. This process led to significant changes in the textural and thermal properties of the functionalized copolymers.

Modification of the TRIM-GMA copolymers with diethylenetriamine results in a considerable decrease in the specific surface area, pore volume and pore diameter. Interestingly, the thermal stability of the polymer increases with the introduction the functional groups. The TG, DTG and DSC curves of parent copolymer are at variance with those of the corresponding functionalized polymers.

Acknowledgements The research leading to these results has received funding from the People Programme (Marie Curie Actions) of the European Union's Seventh Framework Programme FP7/ 2007-2013/under REA grant agreement no PIRSES-GA-2013-612484

Open Access This article is distributed under the terms of the Creative Commons Attribution 4.0 International License (http://crea tivecommons.org/licenses/by/4.0/), which permits unrestricted use, distribution, and reproduction in any medium, provided you give appropriate credit to the original author(s) and the source, provide a link to the Creative Commons license, and indicate if changes were made.

\section{References}

1. Maciejewska M, Osypiuk-Tomasik J. Studies of sorption properties of porous copolymers of 1-vinyl-2-pyrrolidone. J Therm Anal Calorim. 2013;111:1595-601.

2. Maciejewska M, Szajnecki $Ł$, Gawdzik B. Investigation of the surface area and polarity of porous copolymers of maleic anhydride and divinylbenzene. J Appl Polym Sci. 2012;125:300-7.

3. Podkościelna B. New photoluminescent copolymers of naphthalene-2,7-diol dimethacrylate and $\mathrm{N}$-vinyl-2-pyrrolidone: synthesis, characterisation and properties. J Therm Anal Calorim. 2014;116:785-93.

4. Maciejewska M, Osypiuk-Tomasik J. Sorption on porous copolymers of 1-vinyl-2-pyrrolidone-divinylbenzene. J Therm Anal Calorim. 2013;114:749-55.

5. Grochowicz M. Investigation of the thermal behavior of 4-vinylpyridine-trimethylolpropane trimethacrylate copolymeric microspheres. J Therm Anal Calorim. 2014;118:1603-11.

6. Maciejewska M. Influence of the filler on thermal properties of porous VP-TRIM copolymers. J Therm Anal Calorim. 2015;119: 507-13.

7. Kip C, Maras B, Evirgen O, Tuncel A. A new type of monodisperse porous, hydrophilic microspheres with reactive chloroalkyl functionality: synthesis and derivatization properties. Colloid Polym Sci. 2014;292:219-28.

8. Stępnik K, Malinowska I, Maciejewska M. A new application of micellar liquid chromatography in the determination of free ampicillin concentration in the drug-human serum albumin standard solution in comparison with the adsorption method. Tallanta. 2016;153:1-7.
9. Zaleski R, Goworek J, Maciejewska M. Positronium lifetime in porous VP-DVB copolymer. Phys Status Solidi C. 2009;6:2445-7.

10. Van Berkel PM, Van Der Slot SC, Driessen WL, Reedijik J, Sherrington DC. Influence of the polymer matrix on the methalion uptake characteristics of ligand-modified poly(glycidyl methacrylate-co-trimethylolpropane trimethacrylate) polymers. Eur Polym J. 1997;33:301-10.

11. Podkościelna B. Synthesis, modification and porous properties of new glycidyl methacrylate copolymers. J Appl Polym Sci. 2011; 120:3020-6.

12. Ferreira A, Bigan M, Blondeau D. Optimization of a polymeric HPLC phase: poly(glycidyl methacrylate-co-ethylene dimethacrylate): influence of the polymerization conditions on the pore structure of macroporous beads. React Funct Polym. 2003;56:123-36.

13. Miletić N, Rohandi R, Vuković Z, Nastasović A, Loos K. Surface modification of macroporous poly(glycidyl methacrylate-co-ethylene glycol dimethacrylate) resins for improved Candida Antarctica Lipase B immobilization. React Funct Polym. 2009;69:68-75.

14. Hainey P, Sherrington DC. Oligoamine-functionalised poly(glycidyl methacrylateethyleneglycol dimethacrylate) resins as moderate base extractants for gold from cyanide solutions. React Funct Polym. 2000;43:195-210.

15. Labbe A, Brocas AL, Ibarboure E, Ishizone T, Hirao A, Deffieux A, Carlotti S. Selective ring-opening polymerization of glycidyl methacrylate: toward the synthesis of cross-linked (Co)polyethers with thermoresponsive properties. Macromolecules. 2011;44:6356-64.

16. Grama S, Plichta Z, Trchova M, Kovarova J, Benes M, Horak D. Monodisperse macroporous poly(glycidyl methacrylate) microspheres coated with silica: design, preparation and characterization. React Funct Polym. 2014;77:11-7.

17. Drelinkiewicz A, Knapika A, Stanucha W, Sobczak J, Bukowska A, Bukowski W. Diamine functionalized gel-type resin as a support for palladium catalysts: preparation, characterization and catalytic properties in hydrogenation of alkynes. React Funct Polym. 2008;68:1652-64.

18. Švec F, Hrudkova H, Horak D, Kalal J. Reaction of the epoxide groups of the copolymer glycidyl methacrylate-ethylene dimethacrylate with aliphatic amino compaunds. Angew Makromol Chem. 1977;63:23-6.

19. Senkal BF, Bicak N. Glycidyl methacrylate based polymer resins with diethylene triamine tetra acetic acid functions for efficient removal of $\mathrm{Ca}(\mathrm{II})$ and $\mathrm{Mg}(\mathrm{II})$. React Funct Polym. 2001;49: $151-7$.

20. Hercigonja R, Maksin D, Nastasović A, Trifunović S, Glodić P, Onjia A. Adsorptive removal of technetium-99 using macroporous poly(GMA-co-EGDMA) modified with diethylenetriamine. J Appl Polym Sci. 2012;12:1273-81.

21. Podkościelna B, Maciejewska M, Bartnicki A. Studies on synthesis and physicochemical properties of new bis[4-(2-hydroxy-3methacryloyloxypropoxy) phenyl]sulfide terpolymers. J Appl Polym Sci. 2012;123:59-65.

22. Lee J-H, Kim B-S, Lee J-O, Imaoka S, Yashinaga $\mathrm{K}$. Crosslinking of grafted copolymer on colloidal silica and introduction of surface amino group. Polym J. 2007;39:1018-24.

23. Maciejewska M, Bolbukh Y. Study on synthesis and characterization of porous microspheres with pendant amine groups. Adsorpt Sci Technol. 2015;33:6-8.

24. Maciejewska M, Kołodyńska D. Synthesis and characterization of porous microspheres bearing pyrrolidone units. Mater Chem Phys. 2015;149:43-50.

25. Grochowicz M, Gawdzik B. Preparation and characterization of porous crosslinked microspheres of new aromatic methacrylates. J Porous Mater. 2013;20:339-49. 